\title{
Superior sensory, motor, and cognitive performance in elderly individuals with multi-year dancing activities
}

\author{
Jan-Christoph Kattenstroth ${ }^{1}$, Izabella Kolankowska ${ }^{1}$, Tobias Kalisch ${ }^{2}$ and Hubert R. Dinse ${ }^{1 *}$ \\ ' Neural Plasticity Lab, Institute for Neuroinformatics, Ruhr-University Bochum, Bochum, Germany \\ 2Department of Neurology, BG-Kliniken Bergmannsheil, Ruhr-University Bochum, Bochum, Germany
}

Edited by:

Lars Nyberg, Umeå University,

Sweden

Reviewed by:

Fredrik Ullén, Stockholm Brain

Institute, Sweden

Lars Nyberg, Umeå University,

Sweden

${ }^{*}$ Correspondence:

Hubert R. Dinse, Neural Plasticity Lab,

Institute for Neuroinformatics,

Ruhr-University Bochum, D-44780

Bochum, Germany.

e-mail: hubert.dinse@rub.de
Aging is associated with a progressive decline of mental and physical abilities. Considering the current demographic changes in many civilizations there is an urgent need for measures permitting an independent lifestyle into old age. The critical role of physical exercise in mediating and maintaining physical and mental fitness is well-acknowledged. Dance, in addition to physical activity, combines emotions, social interaction, sensory stimulation, motor coordination and music, thereby creating enriched environmental conditions for human individuals. Here we demonstrate the impact of multi-year (average 16.5 years) amateur dancing (AD) in a group of elderly subjects (aged 65-84 years) as compared to education-, gender- and aged-matched controls (CG) having no record of dancing or sporting activities. Besides posture and balance parameters, we tested reaction times, motor behavior, tactile and cognitive performance. In each of the different domains investigated, the AD group had a superior performance as compared to the non-dancer CG group. Analysis of individual performance revealed that the best participants of the AD group were not better than individuals of the CG group. Instead, the AD group lacked individuals showing poor performance, which was frequently observed for the CG group. This observation implies that maintaining a regular schedule of dancing into old age can preserve cognitive, motor and perceptual abilities and prevent them from degradation. We conclude that the far-reaching beneficial effects found in the AD group make dance, beyond its ability to facilitate balance and posture, a prime candidate for the preservation of everyday life competence of elderly individuals.

Keywords: aging, dance, successful aging, plasticity, neurotrophic factors, intervention, seniors, enriched environment

\section{INTRODUCTION}

Aging is associated with a progressive decline of perception, motor behavior, cognition and memory functions (Willis, 1987; Mayer and Baltes, 1996; Krampe, 2002; Dinse, 2006; Persson et al., 2006; Dinse et al., 2009), which is paralleled by a general decline of physical fitness (Buchner et al., 1996; Samson et al., 2000; Singh et al., 2006). Conceivably, under these conditions, the preservation of everyday life competence and thus the maintenance of independent living are at severe risk. In particular, impaired mental and physical capacities increase the risk of falling with massive burden for the individual and the society (Lajoie and Gallagher, 2004; Filiatrault et al., 2009). Therefore, given the dramatic demographic changes of industrialized countries characterized by an increasing probability of reaching old and very old age, there is an urgent need for measures permitting an independent lifestyle into old age. Maintenance of independence in elderly individuals has been linked to the concept of "successful aging", describing an avoidance of disease and disability, a maintenance of high physical and cognitive function, and a sustained engagement in social and productive activities (Rowe and Kahn, 1997). In fact, in healthy elderly individuals there is a close association between physical fitness and cognitive performance (Colcombe et al., 2004; Schaefer et al., 2006; Sumic et al., 2007). Consequently, many studies in elderlies have shown that improving aerobic capacities through physical exercise programs has beneficial effects on cognitive performance (Kramer et al., 1999; Colcombe et al., 2006; Deley et al., 2007; Sumic et al., 2007; Hillman et al., 2008; Voelcker-Rehage et al., 2010).

Animal research on the effects of physical exercise suggests a crucial involvement of brain-derived neurotrophin factors and other nerve growth factors (Neeper et al., 1995; Churchill et al., 2002), which play a fundamental role in control and maintenance of synaptic connections and plasticity. On the other hand, it is now welldocumented that age-related changes are not a simple reflection of degenerative processes, but a complex mix of plastic and adaptive and compensatory mechanisms (Godde et al., 2002; Dinse, 2006; Persson et al., 2006; David-Jürgens et al., 2008), suggesting that brain plasticity is operational into old age. Therefore, new strategies for interventions such as training, exercising, practicing and stimulation, which make use of neuroplasticity principles, have been developed to maintain health and functional independence throughout lifespan. The effectiveness of stimulation and training procedures to ameliorate cortical and behavioral age-related degradation implies that aging effects are not irreversible but treatable (Bock and Schneider, 2002; Sawaki et al., 2003; Dinse, 2005; Dinse et al., 2005, 2006; Mahncke et al., 2006; Boyke et al., 2008; Kalisch et al., 2008, 2010).

Several years ago, we demonstrated that housing aged rats under enriched environmental conditions delayed and ameliorated age-related deterioration of brain organization and sensorimotor 
behavior (Hilbig et al., 2002, 2007; Dinse, 2006). Environmental enrichment of this type is believed to present animals with increased sensory, motor, and cognitive demands thereby enforcing physical exercise together with neuroplasticity processes. Given the fact that enriched environmental housing is a powerful tool to ameliorate age-related changes in rats, we proposed that dance represents an equivalent for enriched environmental conditions in humans since it comprises - beyond requirements for physical activity, rhythmic motor coordination, balance and memory emotions, affection, social interaction, acoustic stimulation, and musical experience. In fact, using positron emission tomography has been shown recently that dancing elicits multisite brain activations (Brown et al., 2006) implicating the involvement of widespread interacting brain networks. Moreover, as dancing can be performed at any level, which encourages regular and continued participation, it has a great appeal to many people resulting in a high compliance with little drop-outs.

For many of these reasons, dance has been established as a therapeutic tool for the treatment of Parkinson's disease (Fallik, 2007; Earhart, 2009; Hackney and Earhart, 2009), dementia (PaloBengtsson and Ekman, 2002; Hokkanen et al., 2008), overweight children (Murphy et al., 2009) and patients with serious mental illness (Hackney and Earhart, 2010).

Most studies employing dancing as an intervention in elderly focused on improvement of cardiovascular parameters, muscle strength and of posture and balance (Hopkins et al., 1990; Estivill, 1995; Adiputra et al., 1996; Crotts et al., 1996; Shigematsu et al., 2002; Federici et al., 2005; Hui et al., 2008; Kreutz, 2008; Zhang et al., 2008; Sofianidis et al., 2009), with few studies addressing cognitive abilities (Verghese, 2006; Alpert et al., 2009). We here went one step further by hypothesizing that long-year dancing activity in an elderly population should promote general advantages including preservation of cognitive, motor and sensorimotor performance as well as perceptual abilities. This assumption was based on the one hand on far-reaching beneficial effects observed in aged rats under enriched environmental conditions, on the other hand, we expected a synergistic action of maintained physical exercise together with the impact of neuroplasticity mechanisms through principles of maintained sensory stimulation and maintained use. We therefore studied the impact of multi-year regular ballroom dancing in a group of neurologically healthy elderly subjects (amateur dancing; $\mathrm{AD}$ ) in comparison to a passive control group (CG). Besides the recording of posture and balance parameters, we extended the assessment to measurements of cognitive, attentional, intellectual, perceptual and sensorimotor performance with the aim to cover basic measures of sensory and motor performance as well as rather complex tasks with higher cognitive demands. We found that in each of the different levels investigated, the $\mathrm{AD}$ group showed a superior performance as compared to the non-dancer CG indicating far-reaching beneficial effects of dancing beyond aspects of balance and posture.

\section{MATERIALS AND METHODS SUBJECTS}

A total of 62 healthy elderly volunteers (61-94 years) participated in our study. Subjects were recruited by advertisements in newspapers, poster-announcements and word-of-mouth advertising and agreed to report their medical history, actual medication and underwent the Mini Mental Status Examination (MMSE) (Folstein et al., 1975), to test for dementia. The amateur dancer group (AD) $(n=24,19$ female, $71.69 \pm 1.15$ years) had a longtime history of amateur dancing ( $16.5 \pm 12.7$ years $)$ with an average performing workload of $1.33 \pm 0.24 \mathrm{~h}$ per week. The non-dancer CG consisted of 38 subjects (71.66 \pm 1.13 years, 30 female) having no record of either dancing or sporting activities. The age-distribution $(p=0.859)$ and education level (number of school years, $p=0.603$ ) of subjects across the groups was balanced. All subjects gave their written informed consent before participating. The study was approved by the local Ethics Committee of the Ruhr-University of Bochum.

\section{EVERYDAY COMPETENCE}

Lifestyle and general activity levels were assessed using the custom-made Everyday Competence Questionnaire" (ECQ, unpublished) addressing aspects of everyday life like independence in activities of daily living and mobility, social relations, general health status and life contentment. The compilation of questions used in the ECQ accounts for changed living conditions of today's seniors. The ECQ consisted of 17 items with one specific question per item characterizing so-called instrumental activities of daily living (IADL) such as housekeeping, daily routine, manual skills, mobility, sports, subjective well-being, linguistic abilities and leisure time activities (Willis, 1987). These domains are not necessary for fundamental functioning, but they let an individual live independently in a community.

All subjects were asked to additionally comment on the questions as detailed as possible thereby allowing insight in their habits and living conditions. The answers were converted in scores according to an item-specific scale. Item 17 ("fluency of speech") was exclusively based on the rating of the experimenter. Altogether subjects could achieve 0 to 54 points. The scores were normalized to a scale from 0 to 1 by dividing the number of points achieved by a subject with the maximum possible scores per item.

\section{COGNITIVE PERFORMANCE}

General intelligence was assessed using the Raven Standard Progressive Matrices (RSPM), a non-reading, non-language based measure of fluid intelligence (Raven, 1938). The test was administered according to standard instructions with a 30-min time constraint. In the CG, the RSPM was conducted in a pre/post design to provide data for a separate study. Therefore, for both groups we here used odd-numbered items only. In addition, the non-verbal geriatric concentration test (AKT) was run to assess selective attention and concentration (Gatterer, 1990). For this paper-and-pencil test, subjects had to mark 20 symbols out of 55 similar looking patterns within a time-limit of $30 \mathrm{~s}$. After an initial training session, three consecutive sessions were run and averaged for evaluating individual performance.

\section{MULTIPLE-CHOICE REACTION TIME MEASUREMENT (RT)}

We performed multiple-choice reaction time measurements in a finger selection visuo-tactile task as adopted from Alegria and Bertelson (1970). Subjects were seated $3 \mathrm{~m}$ in front of a monitor. An image of each hand was displayed on the monitor and 1 finger out of 10 was selected by a visual marker. Subjects had to press the key 
corresponding to the selected finger on a hand-shaped 10 button keyboard as fast as possible. One session consisted of 4 blocks of 100 trials each, which were separated by a short break after each block. Maximum response to stimulus interval for each trial was $2000 \mathrm{~ms}$. Each finger was tested 40 times in random order.

\section{POSTURE, BALANCE AND GAIT CONTROL}

Three tests were used to assess the subjects' ability to control their posture, to maintain balance and to evaluate their security of gait. The Romberg test is a standard neurological test addressing joint position sense (proprioception), and was applied in a condition with eyes either open or closed (Lanska and Goetz, 2000; Goble et al., 2009). The subjects were asked to stand upright with their feet in tandem stance. The movements of the body in relation to a perpendicular object behind the subject were monitored. A second experimenter was standing close to the subject to prevent the person from falling. The time until a subject starts to loose balance was recorded (maximal testing-time was limited to $1 \mathrm{~min}$ ). In the timed upergo test subjects were asked to stand up from a sitting position, walk $3 \mathrm{~m}$, return to the chair and sit down again (Podsiadlo and Richardson, 1991). The time to fulfill the task was measured. In the standing-turn-test a standing subject was asked to perform a $360^{\circ}$ turn (Tinetti, 1986). Time and number of steps were documented.

\section{MOTOR PERFORMANCE}

Hand-arm fine-motor performance was evaluated using a commercial, computer-based test-battery for clinical neuropsychological research (MLS, Dr. G. Schuhfried GmbH, Mödling, Austria) as described previously (Kalisch et al., 2006). The system consists of a work plate with two pencils for left and right hand use. All parts of the system are connected to an interface and a PC computer to record the time and number of errors during different tasks. We investigated speed, accuracy, and maintenance of upper limb position during execution of fine-motor movements of the left and right arm, hand, and fingers using following tests:

\section{Steadiness}

Steadiness evaluates the ability to obtain a prescribed arm-hand position and to maintain it for a defined time period. Subjects were asked to place the pencil into a small circular hole $(5.8 \mathrm{~mm})$ of the horizontally positioned board, and hold it there without touching the edges for $32 \mathrm{~s}$ without support of the hand. This task tests the ability to hold a steady position, and allows an estimate of postural tremor. Dependent variables were the number of errors, i.e., the number of contacts of the pencil with the wall of the hole.

\footnotetext{
Aiming

Aiming evaluates the ability to accomplish fast arm-hand movements for small targets. Subjects had to consecutively hit 20 linearly arranged small contact fields (diameter $5 \mathrm{~mm}$, midpoint separation $9 \mathrm{~mm}$ ) with a test pencil. This test assesses the degree of ataxia and the speed of movement by the ability to make rapid repeated aimed movements. The dependent variables were the number of errors (missed contact fields) and the total time needed to complete the task.
}

\section{Pin plugging}

Pin plugging evaluates fine and gross motor dexterity and coordination. The board carries two rows of 25 small holes, one on the left side and one on the right side. Two containers, each equipped with 25 metal pins, were placed in $30 \mathrm{~cm}$ distance from the right and left side of the board. The subjects were asked to pick the pins with their right hand, one by one, from the right container and insert them into the holes on the peg-board. Subsequently the test was continued using the left hand and left container. If one of the metal pins dropped during the transfer, they were instructed to go on with the next one. Time to complete the test was assessed.

\section{Tapping}

Tapping evaluates the ability to perform very fast, repetitive wristfinger movements with little emphasis on precision of movement. Subjects were required to hit a square contact plate ( 40 by $40 \mathrm{~mm}$ ) on the test board with a test pencil as frequently as possible. The measured parameter was number of hits achieved in a time interval of $32 \mathrm{~s}$, which provides a measure of the speed of antagonistic oscillation. In this task, support of the forearm was allowed. Therefore, the repetitive contacts had to be accomplished by wrist movements.

\section{TACTILE PERFORMANCE Touch threshold}

Touch threshold was evaluated by probing the fingertips with von Frey filaments (Marstocknervtest, Marburg, Germany). Each filament was calibrated to a known buckling force determined by its length and diameter. The test kit contained 16 different filaments calibrated to forces ranging from $0.25-294 \mathrm{mN}$ in logarithmic scaling. Additional two filaments (Touch Test, Stoelting Co, Wood Dale, IL, USA) with forces of $0.08 \mathrm{mN}$ and $0.20 \mathrm{mN}$ were used to expand the effective test range. Fine-touch sensitivity was tested with a staircase procedure, during which subjects were required to indicate whenever they perceived an indentation. The applied contact forces were decreased in a stepwise manner until the subjects no longer perceived the stimulus (lower boundary), and then increased until the stimulus was perceived again (upper boundary). This procedure was repeated three times, resulting in six values that were averaged to form the absolute touch threshold.

\section{Two-point-discrimination threshold (2pd)}

Spatial 2pd thresholds were assessed on the tips of the left (LID) and right (RID) index finger using the method of constant stimuli as described previously (Dinse, 2005; Kalisch et al., 2007, 2008). We tested seven pairs of brass needles; in addition zero distance was tested with a single needle. To overcome problems in the use of two-point measurements associated with hand-held probes, we used a specifically designed apparatus that assure a standardized form of testing (cf. Figures in Dinse et al., 2005, 2006). We used needle separations of $1.5,2.3,3.1,3.9,4.7,5.6$ and $7 \mathrm{~mm}$. The diameter of the needles was $0.7 \mathrm{~mm}$ and the diameter of the blunt endings was $200 \mu \mathrm{m}$. Application-force was about 150 to $200 \mathrm{mN}$. All conditions were presented eight times in randomized order resulting in 64 tests per session. As described previously, test-retest reliability using this procedure was 0.90 for young subjects, and 0.88 for elderly participants (Dinse et al., 2006). Subjects, who were not 
informed about the ratio of needle-pairs and single needles, which was $7: 1$, had to decide immediately if they had the sensation of one or two needles. They were instructed to classify the percept of a single needle or doubtful stimuli as "one" but the distinct percept of two stimuli as "two". The summed responses were plotted against the needle-distances resulting in a psychometric function, which was fitted by a binary logistic regression (SPSS; SPSS Inc., USA). Threshold was taken from the fit where 50\% correct responses were reached.

\section{DOMAINS}

To collapse the data obtained for the various tests, we formed five domains covering similar functional categories. "Cognitive performance" comprised data from the AKT and the RSPM. "Tactile performance" comprised data from touch threshold and $2 p d$. "Posture\&balance" comprised data from the Romberg test, the timed upergo test and the standing-turn test. "Motor performance" comprised Steadiness, Aiming, Pin plugging and Tapping. A separate domain "RT" was introduced to cover data from the multiplechoice reaction time task.

\section{INDICES OF PERFORMANCE (IP)}

To compare performances across all tests and all subjects of both groups we calculated normalized performance indices for each subject and each test as (wp-ip)/(wp-bp), where wp is the worst performance of all subjects, ip is the individual performance, and bp is the best performance of all subjects. Best IP is 1, while worst IP is 0 . Indices were subsequently averaged across tasks belonging to a particular domain as described above.

\section{DATA ANALYSIS}

In all cases we report averages and standard error of the mean (SEM). We hypothesized the AD group to show at least a comparable, or a superior performance in comparison to the CG group. Therefore we used a one-tailed Student's $t$-test to detect differences between the two groups. Alpha levels were adjusted according to Bonferroni's method (23 tests). Moreover, we computed effect sizes according to Cohen's d (Cohen, 1988). For tests of differences in the distribution we used Chi-square statistics. A $p$-value of $<0.05$ was considered significant. In order to detect possible relationships between dance experience and performance, correlation analysis were conducted using Pearson's linear correlation coefficients for total dance experience (years) and average workload (minutes week).

\section{RESULTS}

We tested cognitive, perceptual and motor performance in two groups of age- and education-matched elderly participants who had a long-year record of regular $\mathrm{AD}$ or no dancing experience (control group; CG). We found that the AD group had a superior performance in most of the tests investigated. In Table 1, all mean values $( \pm$ SEM) are shown for each task together with the result of the $t$-test comparing both groups. Performance for individual tests is illustrated in Figure 1.

Everyday competence was assessed using the ECQ. The AD group showed a significantly higher ECQ-score than the CG group. In the tests assessing cognitive performance, the $\mathrm{AD}$ group had higher scores in both the RSPM (Figure 1A) and the AKT. Reaction times obtained for the multiple-choice RT task were averaged for the left and right hand. For both hands, the AD group had faster RTs.

When testing posture and balance we found no differences for the Romberg test, neither for the parameter [eyes open] nor [eyes closed]. In contrast, for the standing-turn test participants of the AD group needed fewer steps for completion. Moreover, the AD group was faster in the timed up\& go task (Figure 1B). In the motor domain, for the subtest Steadiness for the left hand the number of errors was higher in the CG group. The subtest Aiming revealed better performance for the parameter [error] for the right, but not for the left hand. Although significance criteria was missed, in the subtest Pin plugging the AD group was faster with both hands (Figure 1C). Significant differences for the left hand was found for the subtest Tapping, where the AD group reached higher rates. Touch thresholds revealed no differences between groups, but $2 \mathrm{pd}$ thresholds for the index fingers of both hands were lower in the AD group (Figure 1D).

Correlation analyses revealed significant correlations only between individual dance workload per week and $2 \mathrm{pd}$ thresholds of the right index finger (Pearson's $r=0.566, p=0.018$ ), and between individual dance experience (years) and Romberg test performed with eyes closed (Pearson's $r=0.571, p=0.033$ ).

\section{INDICES OF PERFORMANCE (IP)}

The calculation of IP for each test and each subject allowed a direct comparison of performances across all tests and all subjects, and a grouping into functional domains as defined in Section "Materials and Methods". As shown in Table 2 and Figure 2, in all domains, which assemble all tests applied, the AD group showed significant higher IPs, with the largest advantage for posture and balance parameters.

Our findings demonstrated a general advantage for the group of $\mathrm{AD}$, which spans cognitive, perceptual and motor performance. In order to obtain insight into possible differences of the distribution of the individual data-sets, we performed categorical analysis of IP (binned into $>0.5$ and $<0.5$ ). In all of the 5 domains analyzed, the CG group showed a higher occurrence of IPs lower 0.5 (subjects with index $<0.5$, "Cognition": $\mathrm{AD}=11.47 \%, \mathrm{CG}=36.26 \%, \chi^{2}=11.57, p=0.001$; "RTs": $\mathrm{AD}=14.58 \%, \mathrm{CG}=44.74 \%, \chi^{2}=12.086, p=0.001$; "Posture\&balance": $\mathrm{AD}=32.43 \%, \mathrm{CG}=70.37 \%, \chi^{2}=17.99$, $p \leq 0.001$; "Motor performance": $\mathrm{AD}=5.91 \%, \mathrm{CG}=24.59 \%$, $\chi^{2}=38.16, p \leq 0.001$ : "Tactile performance": $\mathrm{AD}=0 \%$, $\left.\mathrm{CG}=30.77 \%, \chi^{2}=25.51, p \leq 0.001\right)$. Accordingly, the overall superior advantage of AD individuals did not come from the fact that their best performers were better than those of the CG group, but because the AD group lacked poor performer as frequently present in the CG group.

\section{DISCUSSION}

We investigated the impact of multi-year AD in a group of 24 elderly subjects, who had an average record of regular dancing of 16.5 years. In addition to posture and balance, which are closely related to dancing, we performed a broad assessment of cognitive, attentional, perceptual and sensorimotor abilities. We found that in each of the different categories investigated, the $\mathrm{AD}$ group showed a superior 
Table 1 | Comparison of cognitive, posture, balance and sensorimotor status of AD and CG.

\begin{tabular}{|c|c|c|c|c|c|c|}
\hline Variables & AD & (Range) & CG & (Range) & $\begin{array}{l}p \text {-value } \\
\text { corrected }\end{array}$ & Effect strength \\
\hline Age [years] & $71.69 \pm 1.15$ & $(65-84)$ & $71.66 \pm 1.13$ & $(61-94)$ & 0.859 & \\
\hline Education level [school years] & $9.88 \pm 0.45$ & $(7-13)$ & $10.16 \pm 0.33$ & $(6-13)$ & 0.603 & \\
\hline Everyday competence (ECQ) & $10.71 \pm 0.36$ & (7.56-13.85) & $8.43 \pm 0.34$ & $(5.04-12.59)$ & $\leq 0.001$ & 1.21 \\
\hline Geriatric concentration test (AKT) & $54.29 \pm 0.13$ & $(52.67-55)$ & $53.49 \pm 0.29$ & $(47-55)$ & 0.590 & 0.59 \\
\hline \multicolumn{7}{|l|}{ REACTION TIMES } \\
\hline Multiple-choice reaction times [ms], L & $690.93 \pm 20.73$ & $(503.35-933.41)$ & $780.30 \pm 19.39$ & (581.01-1081.17) & 0.017 & 0.90 \\
\hline Multiple-choice reaction times [ms], $\mathrm{R}$ & $677.52 \pm 17.89$ & $(518.55-868.24)$ & $760.32 \pm 18.01$ & (580.51-1012.44) & 0.039 & 0.83 \\
\hline \multicolumn{7}{|l|}{ POSTURE\&BALANCE } \\
\hline Standing-turn [s] & $2.39 \pm 0.20$ & $(1.42-4.30)$ & $3.21 \pm 0.33$ & $(1.77-5.09)$ & 0.460 & 0.88 \\
\hline Up\&go [s] & $6.09 \pm 0.24$ & $(4.48-8)$ & $7.42 \pm 0.25$ & (5.54-8.54) & 0.013 & 1.46 \\
\hline \multicolumn{7}{|l|}{ MOTOR PERFORMANCE } \\
\hline \multicolumn{7}{|l|}{ Hand-arm steadiness } \\
\hline Steadiness [error], L & $1.57 \pm 0.86$ & $(0-9)$ & $15.82 \pm 2.49$ & $(0-54)$ & 0.016 & 1.29 \\
\hline Steadiness [error], $\mathrm{R}$ & $3.14 \pm 1.04$ & $(0-12)$ & $13.08 \pm 2.72$ & $(0-71)$ & 0.442 & 0.82 \\
\hline \multicolumn{7}{|l|}{ Control Precision } \\
\hline Aiming [error], L & $1.50 \pm 0.43$ & $(0-6)$ & $1.00 \pm 0.26$ & $(0-5)$ & 1.0 & 0.31 \\
\hline Aiming [error], $\mathrm{R}$ & $0.14 \pm 0.10$ & $(0-1)$ & $1.87 \pm 0.27$ & $(0-6)$ & 0.005 & 1.42 \\
\hline Aiming [s], L & $10.01 \pm 0.96$ & $(0.01-15.84)$ & $11.18 \pm 0.40$ & (7.19-18.37) & 1.0 & 0.38 \\
\hline Aiming [s], R & $9.48 \pm 0.50$ & (6.66-13.74) & $10.66 \pm 0.28$ & $(7.96-17.11)$ & 0.470 & 0.66 \\
\hline \multicolumn{7}{|l|}{ Rate of wrist movement } \\
\hline Touch threshold [mN], RID & $0.27 \pm 0.02$ & $(0.20-0.39)$ & $0.34 \pm 0.04$ & $(0.08-0.94)$ & 1.0 & 0.45 \\
\hline 2-Point-discrimination-threshold [mm], LID & $2.90 \pm 0.07$ & $(2.26-3.30)$ & $3.45 \pm 0.09$ & $(2.20-4.64)$ & 0.003 & 1.30 \\
\hline 2-Point-discrimination-threshold [mm], RID & $3.15 \pm 0.07$ & $(2.77-3.61)$ & $3.59 \pm 0.07$ & (2.89-4.58) & 0.002 & 1.28 \\
\hline
\end{tabular}

$A D$, amateur dancer; $C G$, control group; $L$, left hand; R, right hand; $L I D$, left index finger; RID, right index finger. Values are means, SEM. ${ }^{1}$ Raven Standard Progressive Matrices, subset of 30 items.

performance as compared to the non-dancer CG group. The largest advantage was found for posture and balance parameters, which is in line with previous findings showing beneficial effects of dance on fitness and posture (Hopkins et al., 1990; Estivill, 1995; Adiputra et al., 1996; Crotts et al., 1996; Shigematsu et al., 2002; Kreutz, 2008; Sofianidis et al., 2009).

Falls are a major health problem in an aging society, which arise as a consequence of a decline in postural and balance due to muscle weakness and other risk factors (Melzer et al., 2001; Sturnieks et al., 2008). Falls, or even the fear of falling lead to a reduced quality of life (Voermans et al., 2007). Therefore, various forms of intervention programs have been developed to counteract the risk of falling, many of them employ dancing. On the other hand, attempts have been made to identify specific risk factors. For example, reaction times, which require high attention, fast and well-coordinated motor responses and intact sensation, have been identified as a reliable predictor for the risk of falls (Lajoie and Gallagher, 2004). Our findings are compatible with a critical role of RTs in describing the overall status of elderly individuals, as the AD group showed significantly shorter reaction times than the CG.

To address a possible general decline of motor performance with increasing age besides that related to posture and balance, we measured different parameters of hand-arm functions. Subjects of the $\mathrm{AD}$ group showed an overall better performance for hand-arm steadiness, control precision and wrist movements. While some aspects of maintained hand-arm function might be directly related to dancing activity such as preserved muscle strength and sensorimotor coordination, other more unspecific factors such as attention 


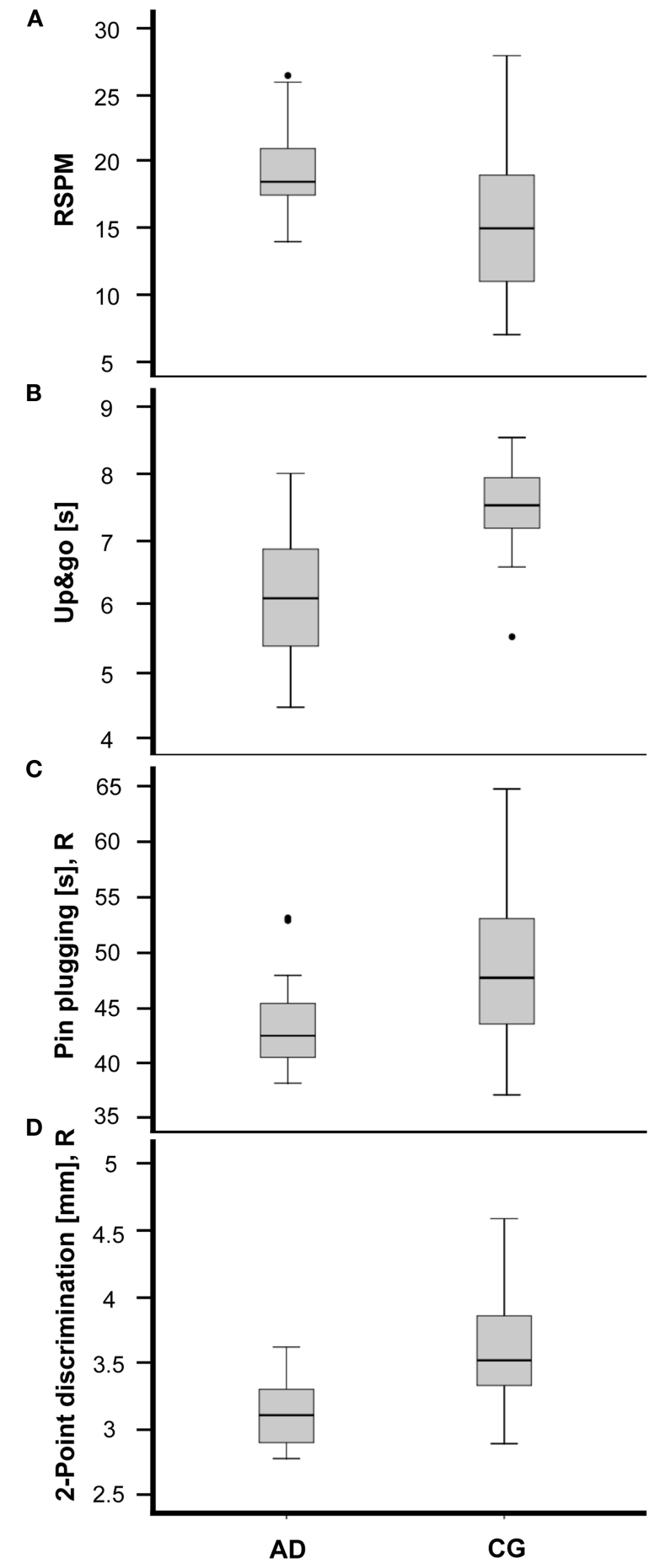

FIGURE 1 | Performance of amateur dancers (AD) and a matched control group (CG) for selected tests covering cognitive, posture\&balance, motor and tactile domains. Participants of the AD group showed (A) higher scores in the RSPM ( $p=0.015)$, (B) shorter Up\&go times $(p=0.013)$, (C) shorter task completion times $(p=0.305)$ in the Pin plugging test (right hand), and (D) lower 2-Point-discrimination threshold (right index finger) $(p=0.002)$. The horizontal lines within the boxes represent the medians. Boxes show the top and bottom quartiles and whiskers represent the minima and maxima within 1.5 interquartile range (IQR). Outliers (>3.0 IQR) are labeled as solid dots. and concentration might also play an important role, particularly for tasks requiring steadiness. In fact, the group of $\mathrm{AD}$ showed a better performance in the geriatric concentration test.

Superior posture and balance can be linked to the requirements imposed by dancing, which is to some extent also true to superior hand-arm function as discussed above. In contrast, tactile abilities appear rather unrelated to dancing. For example, enhanced tactile discrimination abilities found in blind Braille readers have been associated with the unusual and extensive use of the fingers to gather fine-scale spatial tactile information (Van Boven et al., 2000; Goldreich and Kanics, 2003). Similarly, tactile acuity in professional pianists is significantly higher as compared to non-musicians, which has been attributed to the extreme usage of the fingers during piano playing (Ragert et al., 2004). We therefore suggest that the superior $2 \mathrm{pd}$ performance found in the $\mathrm{AD}$ group might reflect unspecific factors that are independent of dancing activities and beyond the framework of use. Interestingly, in a recent study of experienced adult Tai Chi practitioners superior spatial tactile acuity in comparison to matched controls has been reported (Kerr et al., 2008). This has been explained by assuming that either individuals with a high fitness are drawn to Tai Chi, or that Tai Chi itself drives cortical changes which lead to superior tactile acuity. We here suggest that the enhanced tactile discrimination performance arises as a consequence of increased levels of neurotrophins, which are upregulated during dancing, and which might also be up-regulated in Tai Chi practitioners.

The fact that both $\mathrm{AD}$ and $\mathrm{CG}$ groups did not differ in respect to absolute touch threshold is compatible with recent observations in elderly individuals according to which tactile acuity can be improved by repetitive sensory stimulation procedures, while absolute touch thresholds remain unchanged (Dinse et al., 2005, 2006). This observation has been taken as an argument that tactile acuity can be improved through changes of synaptic efficacy and synaptic connections, and therefore is subject to neuroplasticity mechanisms. In contrast, touch thresholds seem to reflect predominantly peripheral factors such as mechanoreceptor density and mechanoreceptor composition, which are independent of cortical plasticity processes.

Use-dependent plasticity, synaptic efficacy and the maintenance of synaptic connections are controlled and modulated by neurotrophins, such as brain-derived neurotrophic factor (BDNF). BDNF levels are increased by many factors such as physical activity and social interaction (Neeper et al., 1995; Churchill et al., 2002; Kramer et al., 2006; Vaynman and Gomez-Pinilla, 2006). In particular, housing animals under enriched environmental conditions has been shown to increase neurotrophine gene expression and thus exerting neuroprotective functions (Young et al., 1999; Pham et al., 2002; Mattson et al., 2004). More generally, mild stress response in cells has been advocated as a major driving source up-regulating stress resistance genes and growth factors (Mattson, 2008). Interestingly, among the factors inducing mild stress are sensory stimulation, physical activity and cognitive challenges, all of them involved in dancing.

The overall superior performance of the $\mathrm{AD}$ group in comparison to the controls is highly compatible with these constraints, and with the well-documented positive effects induced by physical activities 
Table 2 | Indices of performance (IP) averaged across individual tasks describing cognition, reaction times, posture\&balance, and motor and tactile performance for both groups.

\begin{tabular}{lllllll}
\hline Domain & AD & (Range) & CG & (Range) & p-value corrected & Effect strength \\
\hline Cognitive performance & $0.76 \pm 0.03$ & $(0.33-1)$ & $0.61 \pm 0.04$ & $(0-1)$ & 0.010 & 0.58 \\
Reaction time & $0.69 \pm 0.03$ & $(0.26-1)$ & $0.52 \pm 0.02$ & $(0-0.87)$ & 0.003 & 0.87 \\
Posture\&balance & $0.68 \pm 0.02$ & $(0.08-1)$ & $0.37 \pm 0.04$ & $(0-1)$ & $\leq 0.001$ & 1.28 \\
Motor performance & $0.81 \pm 0.01$ & $(0-1)$ & $0.70 \pm 0.01$ & $(0-1)$ & 0.012 & 0.47 \\
Tactile performance & $0.75 \pm 0.01$ & $(0.51-1)$ & $0.60 \pm 0.02$ & $(0-1)$ & 0.005 & 0.80 \\
\hline
\end{tabular}

$A D$, amateur dancer; $C G$, control group; Values are means, SEM.

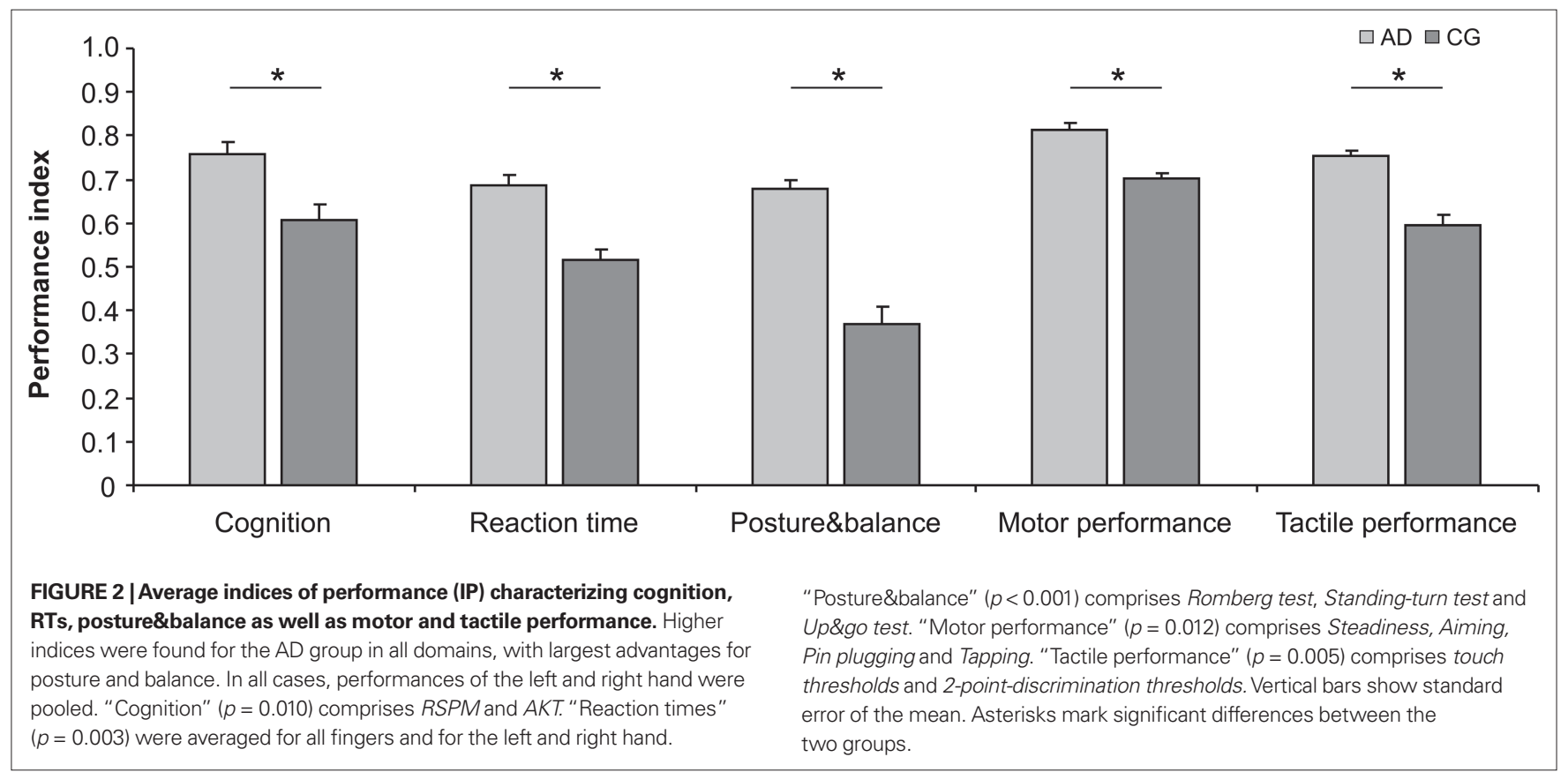

(Kramer et al., 1999, 2006; Vaynman and Gomez-Pinilla, 2006; Deley et al., 2007; Sumic et al., 2007; Hillman et al., 2008; Voelcker-Rehage et al., 2010). It is noteworthy that besides the aspects of physical exercise and the requirements for fine-motor coordination, posture and balance, the emotional aspects of dancing and its close association to music might add further beneficial effects (cf. Gazzaniga, 2008). Studies in children demonstrated that intensive music training was associated with improved performance in the core mathematical system for representing abstract geometry indicating a fundamental association between musical and mathematical cognition (Spelke, 2008). Similarly, learning to dance by effective observation appeared to be closely related to learning by physical practice, both in the level of achievement and also the neural substrates that support the organization of complex actions (Cross et al., 2009). Conceivably, other cognitive skills might benefit from effective observational learning typically associated with dancing.

The analysis of IP, which were calculated to allow direct comparison of performance across all individual tests, provided insight into the question why the $\mathrm{AD}$ group was characterized by a general better performance throughout all categories investigated. We found that individuals of the $\mathrm{AD}$ group were not characterized by an absolutely better performance than that observed in individuals of the $\mathrm{CG}$ group. Instead, the $\mathrm{AD}$ group lacked individuals showing poor performance, which was frequently found for the CG group. This observation implies that maintaining an active lifestyle into old age can preserve cognitive, motor and perceptual abilities and prevent them from degradation, but might not drive levels of performance beyond those typically found in aged populations.

Our data showed that the $\mathrm{AD}$ group reached higher scores in everyday competence as assessed by the ECQ questionnaire which addressed aspects of independence in activities of daily living and mobility, social relations, general health status and life contentment. These findings are in line with a previous study investigating the role of cultural activities, including music, singing and dance for health and life style (Kreutz, 2008). According to this study, dancers were characterized by high-level education and socio-economic status, and their motivation for regular dancing was predominantly driven by both hedonistic and social factors. This raises the question whether the observed superior performance of the AD group was in fact evoked by dancing, or weather a subpopulation characterized by unusual high fitness choose an active lifestyle during early 
adulthood that included dancing, and is able to maintain such a lifestyle over many years. Recent data from an intervention study in a pre-post design showed that after a 6-months dance course elderly participants improved in all aspects tested similar to those described here including perception and cognition (Kattenstroth et al., 2009). These data suggest that dancing in fact plays a crucial role in mediating wide-range beneficial effects. On the other hand, a correlation analysis between outcome measures and the weekly workload as well as the total amount of dancing experience in years revealed significant relationships only for one balance parameter (total amount of dancing experience) and for sensory discrimination thresholds (weekly workload). While this might imply a limited causal role of dancing in mediating positive effects, our recent intervention study demonstrated substantial benefits after a few months of intervention. As a result, potential long-term effects from years of dancing might have saturated resulting in a lack of correlation between outcome measures and amount of dancing experience. Conceivably, both individual predispositions and dancing might play a role in the maintenance of perceptual and cognitive abilities in old age. Apparently, further studies are needed to disentangle their possible contributions.

\section{REFERENCES}

Adiputra, N., Alex, P., Sutjana, D., Tirtayasa, K., and Manuaba,A. (1996). Balinese dance exercises improve the maximum aerobic capacity. J. Hum. Ergol. (Tokyo) 25, 25-29.

Alegria, J., and Bertelson, P. (1970). Time uncertainty, number of alternatives and particular signal-response pair as determinantof choice reaction time. Acta Psychol. 33, 36-44.

Alpert, P., Miller, S., Wallmann, H., Havey, R., Cross, C., Chevalia, T., Gillis, C., and Kodandapari, K. (2009). The effect of modified jazz dance on balance, cognition, and mood in older adults. J. Am. Acad. Nurse Pract. 21, 108-115.

Bock, O., and Schneider, S. (2002). Sensorimotor adaptation in young and elderly humans. Neurosci. Biobehav. Rev. 26, 761-767.

Boyke, J., Driemeyer, J., Gaser, C., Büchel, C., and May, A. (2008). Traininginduced brain structure changes in the elderly. J. Neurosci. 28, 7031-7035.

Brown, S., Martinez, M., and Parsons, L. (2006). The neural basis of human dance. Cereb. Cortex 16, 1157-1167.

Buchner, D., Larson, E., Wagner, E., Koepsell, T., and de Lateur, B. (1996). Evidence for a non-linear relationship between leg strength and gait speed. Age Ageing 25, 386-391.

Churchill, J., Galvez, R., Colcombe, S., Swain, R., Kramer, A., and Greenough, W. (2002). Exercise, experience and the aging brain. Neurobiol. Aging 23, 941-955.

Cohen, J. (1988). Statistical Power Analysis for the Behavioral Science. Hillsdale, NJ: Lawrence Erlbaum Associates, Inc.

Colcombe, S., Erickson, K., Scalf, P., Kim, J., Prakash, R., McAuley, E., Elavsky,
S., Marquez, D., Hu, L., and Kramer, A. (2006). Aerobic exercise training increases brain volume in aging humans. J. Gerontol. A Biol. Sci. Med. Sci. 61, 1166-1170.

Colcombe, S., Kramer, A., Erickson, K., Webb, A., Jerome, G., Marquez, D., and Elavsky, S. (2004). Cardiovascular fitness, cortical plasticity, and aging. Proc. Natl. Acad. Sci. U.S.A. 101, 3316-3321.

Cross, E., Kraemer, D., Hamilton, A., Kelley, W., and Grafton, S. (2009). Sensitivity of the action observation network to physical and observational learning. Cereb. Cortex 19, 315-326.

Crotts, D., Thompson, B., Nahom, M., Ryan, S., and Newton, R. (1996). Balance abilities of professional dancers on select balance tests. J. Orthop. Sports Phys. Ther. 23, 12-17.

David-Jürgens, M., Churs, L., Berkefeld, T., Zepka, R., and Dinse, H. (2008). Differential effects of aging on foreand hindpaw maps of rat somatosensory cortex. PLOS ONE 3, e3399. doi: 10.1371/journal.pone.0003399.

Deley, G., Kervio, G., Van Hoecke, J., Verges, B., Grassi, B., and Casillas, J. (2007). Effects of a one-year exercise training program in adults over 70 years old: a study with a control group. Aging Clin. Exp. Res. 19, 310-315.

Dinse, H. R. (2005). Treating the aging brain: cortical reorganization and behavior. Acta Neurochir. Suppl. 93, 79-84.

Dinse, H. R. (2006). Cortical reorganization in the aging brain. Prog. Brain Res. 157, 57-80.

Dinse, H. R., Kalisch, T., Ragert, P., Pleger, B., Schwenkreis, P., and Tegenthoff, Scalf, P., McAuley, E., Cohen, N.,

Compared to activities such as exercising, walking or playing an instrument, dance has the advantage to combine many diverse features including physical activity, social and emotional interaction, each of them well-documented to have beneficial effects. This unique property might be one reason for its general acceptance and its high compliance. Our study provides strong evidence that dance promotes a wide-range of beneficial effects that are not limited to motor behavior, posture and balance, but covers also perceptual and cognitive abilities. Therefore, dance might be an appropriate approach for enforcing and maintaining plasticity processes in elderly populations, thereby contributing to successful aging.

\section{ACKNOWLEDGMENT}

Parts of the work have been supported by a grant from the Deutsche Forschungsgemeinschaft to HRD (DFG Di 334/10-4). Jan-Christoph Kattenstroth is a recipient of a stipend from the Allgemeiner Deutscher Tanzlehrerverband (ADTV). We thank Peter Hühner from the Tanzstudio Gödde, Dortmund, Germany and Annegret Manek of the Begegnungszentrum DortmundScharnhorst for providing space and support.

M. (2005). Improving human haptic performance in normal and impaired human populations through unattended activation-based learning. ACM Trans. Appl. Percept. 2, 71-88.

Dinse, H. R., Kleibel, N., Kalisch, T., Ragert, P., Wilimzig, C., and Tegenthoff, M. (2006). Tactile coactivation resets agerelated decline of human tactile discrimination. Ann. Neurol. 60, 88-94.

Dinse, H. R., Tegenthoff, M., Heinisch, C., and Kalisch, T. (2009). "Ageing and touch," in The Sage Encyclopedia of Perception, ed. B. Goldstein (London: Sage), 21-24.

Earhart, G. (2009). Dance as therapy for individuals with Parkinson disease. Eur. J. Phys. Rehabil. Med. 45, 231-238.

Estivill, M. (1995). Therapeutic aspects of aerobic dance participation. Health Care Women Int. 16, 341-350.

Fallik, D. (2007). Finding new life through movement: how a modern dance company helps Parkinson's disease patients loosen tight joints and lift spirits. Neurology 3, 30-33.

Federici, A., Bellagamba, S., and Rocchi, M. (2005). Does dance-based training improve balance in adult and young old subjects? A pilot randomized controlled trial. Aging Clin. Exp. Res. 17, 385-389.

Filiatrault, J., Desrosiers, J., and Trottier, L. (2009). An exploratory study of individual and environmental correlates of fear of falling among communitydwelling seniors. J. Aging Health 21, 881-894.

Folstein, M. F., Folstein, S.E., and McHugh, P. R. (1975). "Mini-mental state": a practical method for grading the cognitive state of patients for the clinician. J. Psychiatr. Res. 12, 189-198.
Gatterer, G. (1990). Alters-Konzentrations Test (AKT). Handanweisung. Göttingen: Hogrefe.

Gazzaniga, M. (2008). Learning, Arts, and the Brain: The Dana Consortium Report on Arts and Cognition. New York, Washington, DC: Dana Press.

Goble, D., Coxon, J., Wenderoth, N., Van Impe, A., and Swinnen, S. (2009). Proprioceptive sensibility in the elderly: degeneration, functional consequences and plastic-adaptive processes. Neurosci. Biobehav. Rev. 33, 271-278.

Godde, B., Berkefeld, T., David-Jurgens, M., and Dinse, H. R. (2002). Agerelated changes in primary somatosensory cortex of rats: evidence for parallel degenerative and plasticadaptive processes. Neurosci. Biobehav. Rev. 26, 743-752.

Goldreich, D., and Kanics, I. M. (2003). Tactile acuity is enhanced in blindness. J. Neurosci. 23, 3439-3445.

Hackney, M., and Earhart, G. (2009). Effects of dance on movement control in Parkinson's disease: a comparison of Argentine tango and American ballroom. J. Rehabil. Med. 41, 475-481.

Hackney, M., and Earhart, G. (2010) Social partnered dance for people with serious and persistent mental illness: a pilot study. J. Nerv. Ment. Dis. 198, 76-78.

Hilbig, H., Beier, V., Köster, K., Stoll, C., Richter, D., and Dinse, H. (2007). Enriched environmental conditions ameliorate age-dependent alterations in the somatosensory system, but do not affect the motor system of the rat spinal cord. Neuroembryol. Aging 4, 138-146. 
Hilbig, H., Bidmon, H. J., Steingruber, S., Reinke, H., and Dinse, H. R. (2002). Enriched environmental conditions reverse age-dependent gliosis and losses of neurofilaments and extracellular matrix components but do not alter lipofuscin accumulation in the hindlimb area of the aging rat brain. $J$. Chem. Neuroanat. 23, 199-209.

Hillman, C., Erickson, K., and Kramer, A. (2008). Be smart, exercise your heart: exercise effects on brain and cognition. Nat. Rev. Neurosci. 9, 58-65.

Hokkanen, L., Rantala, L., Remes, A., Härkönen, B., Viramo, P., and Winblad, I. (2008). Dance and movement therapeutic methods in management of dementia: a randomized, controlled study. J. Am. Geriatr. Soc. 56, 771-772.

Hopkins, D., Murrah, B., Hoeger, W., and Rhodes, R. (1990). Effect of lowimpact aerobic dance on the functional fitness of elderly women. Gerontologist 30, 189-192.

Hui, E., Chui, B., and Woo, J. (2008). Effects of dance on physical and psychological well-being in older persons. Arch. Gerontol. Geriatr. 49, e45-e50.

Kalisch, T., Tegenthoff, M., and Dinse, H. R. (2007). Differential effects of synchronous and asynchronous multifinger coactivation on human tactile performance. BMC Neurosci. 8, 58. doi: 10.1186/1471-2202-8-58.

Kalisch, T., Tegenthoff, M., and Dinse, H. R. (2008). Improvement of sensorimotor functions in old age by passive sensory stimulation. Clin. Interv. Aging 3, 673-690.

Kalisch, T., Tegenthoff, M., and Dinse, H. R. (2010). Repetitive electric stimulation elicits enduring improvement of sensorimotor performance in seniors. Neural Plast. 2010, 11.

Kalisch, T., Wilimzig, C., Kleibel, N., Tegenthoff, M., and Dinse, H. R. (2006). Age-related attenuation of dominant hand superiority. PLoS ONE 1, e90. doi: 10.1371/journal. pone. 0000090 .

Kattenstroth, J. C., Kalisch, T., Holt, S. K., Tegenthoff,M., and Dinse,H. R. (2009). Beneficial effects of a six-months dance class on sensorimotor and cognitive performance of elderly individuals. 180.8. In Neuroscience Meeting Planner, Society for Neuroscience. Chicago, IL.

Kerr, C., Shaw, J., Wasserman, R., Chen, V., Kanojia, A., Bayer, T., and Kelley, J. (2008). Tactile acuity in experienced Tai Chi practitioners: evidence for use dependent plasticity as an effect of sensory-attentional training. Exp. Brain Res. 188, 317-322.

Kramer, A., Erickson, K., and Colcombe, S. (2006). Exercise, cognition, and the aging brain. J. Appl. Physiol. 101, 1237-1242.
Kramer, A. F., Hahn, S., Cohen, N. J., Banich, M. T., McAuley, E., Harrison, C. R., Chason, J., Vakil, E., Bardell, L., Boileau, R. A., and Colcombe, A. (1999). Ageing, fitness and neurocognitive function. Nature 400, 418-419.

Krampe, R. T. (2002). Aging, expertise and fine motor movement. Neurosci. Biobehav. Rev. 26, 769-776.

Kreutz, G. (2008). Does partnered dance promote health? The case of tango Argentino. J. R. Soc. Promot. Health $128,79-84$.

Lajoie, Y., and Gallagher, S. (2004). Predicting falls within the elderly community: comparison of postural sway, reaction time, the Berg balance scale and the Activities-specific Balance Confidence $(\mathrm{ABC})$ scale for comparing fallers and non-fallers. Arch. Gerontol. Geriatr. 38, 11-26.

Lanska, D., and Goetz, C. (2000). Romberg's sign: development, adoption, and adaptation in the 19th century. Neurology 55, 1201-1206.

Mahncke, H., Connor, B., Appelman, J., Ahsanuddin, O., Hardy, J., Wood, R., Joyce, N., Boniske, T., Atkins, S., and Merzenich, M. (2006). Memory enhancement in healthy older adults using a brain plasticity-based training program: a randomized, controlled study. Proc. Natl. Acad. Sci. U.S.A. 103, 12523-12528.

Mattson, M. (2008). Hormesis defined. Ageing Res. Rev. 7, 1-7.

Mattson, M., Duan, W., Wan, R., and Guo, Z. (2004). Prophylactic activation of neuroprotective stress response pathways by dietary and behavioral manipulations. NeuroRx 1, 111-116.

Mayer, K. U., and Baltes, P. B. (1996). Die Berliner Altersstudie. Berlin: Akademie Verlag.

Melzer, I., Benjuya, N., and Kaplanski, J. (2001). Age-related changes of postural control: effect of cognitive tasks. Gerontology 47, 189-194.

Murphy, E., Carson, L., Neal, W., Baylis, C., Donley, D., and Yeater, R. (2009). Effects of an exercise intervention using Dance Dance Revolution on endothelial function and other risk factors in overweight children. Int. J. Pediatr. Obes. 4, 205-214.

Neeper, S. A., Gomez-Pinilla, F., Choi, J., and Cotman, C. (1995). Exercise and brain neurotrophins. Nature 373, 109.

Palo-Bengtsson, L., and Ekman, S. (2002). Emotional response to social dancing and walks in persons with dementia. Am. J. Alzheimers Dis. Other Demen. 17, 149-153.

Persson, J., Nyberg, L., Lind, J., Larsson, A., Nilsson, L. G., Ingvar, M., and Buckner, R. L. (2006). Structure-function correlates of cognitive decline in aging. Cereb. Cortex 16, 907-915.
Pham, T., Winblad, B., Granholm,A., and Mohammed,A.(2002).Environmental influences on brain neurotrophins in rats. Pharmacol. Biochem. Behav. 73, 167-175.

Podsiadlo, D., and Richardson, S. (1991). The timed "Up \& Go": a test of basic functional mobility for frail elderly persons. J. Am. Geriatr. Soc. 39, 142-148.

Ragert, P., Schmidt, A., Altenmuller, E., and Dinse, H. R. (2004). Superior tactile performance and learning in professional pianists: evidence for meta-plasticity in musicians. Eur. J. Neurosci. 19, 473-478.

Raven, J. C. (1938). Progressive Matrices. London: Lewis and Co.

Rowe, J., and Kahn, R. (1997). Successful aging. Gerontologist 37, 433-440.

Samson, M., Meeuwsen, I., Crowe, A., Dessens, J., Duursma, S., and Verhaar, H. (2000). Relationships between physical performance measures, age, height and body weight in healthy adults. Age Ageing 29, 235-242.

Sawaki, L., Yaseen, Z., Kopylev, L., and Cohen, L. G. (2003). Age-dependent changes in the ability to encode a novel elementary motor memory. Ann. Neurol. 53, 521-524.

Schaefer, S., Huxhold, O., and Lindenberger, U. (2006). Healthy mind in healthy body? A review of sensorimotor-cognitive interdependencies in old age. Eur. Rev. Aging Phys. Activ. 3, 45-54.

Shigematsu, R., Chang, M., Yabushita, N., Sakai, T., Nakagaichi, M., Nho, H., and Tanaka, K. (2002). Dance-based aerobic exercise may improve indices of falling risk in older women. Age Ageing 31, 261-266.

Singh, A., Chin A Paw, M., Bosscher, R., and van Mechelen, W. (2006). Crosssectional relationship between physical fitness components and functional performance in older persons living in long-term care facilities. BMC Geriatr. 6,4 .

Sofianidis, G., Hatzitaki, V., Douka, S., and Grouios, G. (2009). Effect of a 10 -week traditional dance program on static and dynamic balance control in elderly adults. J. Aging Phys. Act. $17,167-180$.

Spelke, E. (2008). "Effects of music instruction on developing cognitive systems," in Learning, Arts, and the Brain eds C. Asbury and B. Rich (New York/Washington: Dana Press), 17-50.

Sturnieks, D., St George, R., and Lord, S. (2008). Balance disorders in the elderly. Neurophysiol. Clin. 38 , 467-478.

Sumic, A., Michael, Y., Carlson, N., Howieson, D., and Kaye, J. (2007). Physical activity and the risk of dementia in oldest old. J. Aging Health 19, 242-259.

Tinetti, M. (1986). Performance-oriented assessment of mobility problems in elderly patients. J. Am. Geriatr. Soc. 34, 119-126.

Van Boven, R. W., Hamilton, R. H., Kauffman, T., Keenan, J. P., and Pascual-Leone, A. (2000). Tactile spatial resolution in blind braille readers. Neurology 54, 2230-2236.

Vaynman, S., and Gomez-Pinilla, F. (2006). Revenge of the "sit": how lifestyle impacts neuronal and cognitive health through molecular systems that interface energy metabolism with neuronal plasticity.J. Neurosci. Res. 84, 699-715.

Verghese, J. (2006). Cognitive and mobility profile of older social dancers. $J$. Am. Geriatr. Soc. 54, 1241-1244.

Voelcker-Rehage, C., Godde, B., and Staudinger, U. (2010). Physical and motor fitness are both related to cognition in old age. Eur. J. Neurosci. 31, 167-176.

Voermans, N., Snijders, A., Schoon, Y., and Bloem, B. (2007). Why old people fall (and how to stop them).Pract. Neurol. 7, 158-171.

Willis, S. (1987). Cognitive training and everyday competence. Annu. Rev. Gerontol. Geriatr. 7, 159-188.

Young, D., Lawlor, P., Leone, P., Dragunow, M., and During, M. (1999). Environmental enrichment inhibits spontaneous apoptosis, prevents seizures and is neuroprotective. Nat. Med. $5,448-453$.

Zhang, J., Ishikawa-Takata, K., Yamazaki, H., Morita, T., and Ohta, T. (2008). Postural stability and physical performance in social dancers. Gait Posture 27, 697-701.

Conflict of Interest Statement: One Author (Jan-Christoph Kattenstroth) is a recipient of a stipend from the Allgemeine Deutsche Tanzlehrerverband (ADTV).

Received: 02 April 2010; paper pending published: 24 May 2010; accepted: 02 July 2010; published online: 21 July 2010.

Citation: Kattenstroth J-C, Kolankowska I, Kalisch T and Dinse HR (2010) Superior sensory, motor, and cognitive performance in elderly individuals with multi-year dancing activities. Front. Ag. Neurosci. 2:31. doi: 10.3389/fnagi.2010.00031

Copyright (c) 2010 Kattenstroth, Kolankowska, Kalisch and Dinse. This is an open-access article subject to an exclusive license agreement between the authors and the Frontiers Research Foundation, which permits unrestricted use, distribution, and reproduction in any medium, provided the original authors and source are credited. 